\title{
Perfil de tratamento com medicamentos contínuos em pacientes oncológicos
}

\author{
Profile of continuous medical treatment in oncologic patient \\ Perfil del tratamiento farmacológico continuo en pacientes oncológicos
}

Recebido: 26/04/2021 | Revisado: 05/05/2021 | Aceito: 13/05/2021 | Publicado: 31/05/2021

Camile Lorenz

ORCID: https://orcid.org/0000-0003-0973-3385 Universidade Regional do Noroeste do Estado do Rio Grande do Sul, Brasil E-mail: camilelorenz@gmail.com

Sandra Drews Montagner

ORCID: https://orcid.org/0000-0002-2141-0063 Universidade Regional do Noroeste do Estado do Rio Grande do Sul, Brasil E-mail: sandrinhadrews@yahoo.com.br

Eniva Miladi Fernandes Stumm

ORCID: https://orcid.org/0000-0001-6169-0453 Universidade Regional do Noroeste do Estado do Rio Grande do Sul, Brasil E-mail: eniva@unijui.edu.br

Jose Antônio Gonsales da Silva

ORCID: https://orcid.org/0000-0002-9335-2421

Universidade Regional do Noroeste do Estado do Rio Grande do Sul, Brasil E-mail: jose.gonzales@unijui.edu.br

Lenara Schalanski Krause

ORCID: https://orcid.org/0000-0001-6127-8899 Universidade Regional do Noroeste do Estado do Rio Grande do Sul, Brasil E-mail: lenara.krause@ @ou.unijui.edu.br

Juliana Maria Fachinetto

ORCID: https://orcid.org/0000-0002-0864-9643 Universidade Regional do Noroeste do Estado do Rio Grande do Sul, Brasil E-mail: juliana.fachinetto@unijui.edu.br

Christiane Colet

ORCID: https://orcid.org/0000-0003-2023-5088 Universidade Regional do Noroeste do Estado do Rio Grande do Sul, Brasil E-mail:christiane.colet@unijui.edu.br

\begin{abstract}
Resumo
Objetivo: Descrever o perfil de pacientes oncológicos, no início do tratamento, em uso de medicamentos contínuos, assistidos em um CACON. Método: Trata-se de um estudo transversal, quantitativo e analítico. A amostra constituiuse por pacientes com diagnóstico de câncer inseridos para iniciar o tratamento oncológico junto ao Centro de Alta Complexidade (CACON), avaliados no período de agosto de 2018 a janeiro de 2019. Resultados: Participaram da pesquisa 270 pacientes, $53 \%$ do sexo feminino e $58,1 \%$ com mais de 60 anos de idade. Verificou-se associação entre uso de medicamentos e: sexo feminino ( $\mathrm{p}=0,025)$; ser casado ou ter acompanhante $(\mathrm{p}=0,049)$; e possuir mais de 60 anos $(\mathrm{p}=0,000)$. $\mathrm{O}$ grupo de medicamentos mais utilizado pelos pacientes oncológicos em tratamento com medicamentos contínuos foi do aparelho cardiovascular $(69,56 \%)$. Verificada associação entre usar medicamentos contínuos e autorrelato de: hipertensão $(\mathrm{p}=0,000)$; diabetes mellitus $(\mathrm{p}=0,013)$; infarto agudo do miocárdio $(\mathrm{p}=0,045)$; depressão $(\mathrm{p}=0,001)$ e uso de bebidas alcóolicas $(\mathrm{p}=0,000)$. Conclusão: O estudo permitiu conhecer o perfil de uso de medicamentos contínuos e as variáveis associadas, estes dados são fundamentais para propor medidas que melhorem a efetividade e segurança da quimioterapia e dos demais medicamentos associados.
\end{abstract}

Palavras-chave: Medicamentos; Câncer; Doenças crônicas; Paciente.

\section{Abstract}

Goal: Describe the profile of continuous medical treatment in oncologic patient assisted in a CACON. Method: A Crosssectional study, analytical and quantitative. The sample is constituted by patients diagnosed with cancer, inserted to begin oncologic treatment at the High Complexity Center (CACON), evaluated in the period of August, 2018 and January, 2019. Results: Two hundred seventy patients participated in the research, fifty- three percent were of female gender and fifty-one point one percent over sixty years old. It was found asssociation between the use of the medicament and: female sex $(\mathrm{p}=0.025)$; be married or have a companion $(\mathrm{p}=0.049)$; and over sixty years old $(\mathrm{p}=0.000)$. The most used medicament group by oncologic patients in treatment with continuous medicine was the cardiovascular system $(69.56 \%)$. It was verified the association between using continuous medicine and self-reporting of: hypertension $(\mathrm{p}=0.000)$; diabetes mellitus $(\mathrm{p}=0.013)$; acute myocardial infarction $(\mathrm{p}=0.045)$; depression $(\mathrm{p}=0.001)$ and alcoholic 
drinks ( $\mathrm{p}=0.000$ ). Conclusion: It is important to know the treatment profile of the continuous medicine users in oncologic treatment to ensure the patients safety and the effectiveness of the treatment.

Keywords: Medicine; Cancer; Chronic disease; Patient.

\begin{abstract}
Resumen
Objetivo: Describir el perfil de los pacientes oncológicos, al inicio del tratamiento, con medicación continua, asistidos en un CACON. Método: Se trata de un estudio transversal, cuantitativo y analítico. La muestra estuvo constituida por pacientes diagnosticados de cáncer insertados para iniciar tratamiento oncológico en el Centro de Alta Complejidad (CACON), evaluados entre agosto de 2018 y enero de 2019. Resultados: participaron en la investigación 270 pacientes, $53 \%$ de ellos mujeres y 58,1\% mayores de 60 años de edad. Hubo asociación entre el uso de medicamentos y: sexo femenino ( $\mathrm{p}=0.025)$; estar casado o tener pareja $(\mathrm{p}=0,049)$; y tener más de 60 años $(\mathrm{p}=0,000)$. El grupo de fármacos más utilizado por los pacientes oncológicos sometidos a medicación continua fue el sistema cardiovascular $(69,56 \%)$. Se encontró una asociación entre el uso de medicación continua y el autoinforme de: hipertensión $(p=0,000)$; diabetes mellitus ( $\mathrm{p}=0,013)$; infarto agudo de miocardio $(\mathrm{p}=0,045)$; depresión $(\mathrm{p}=0,001)$ y consumo de bebidas alcohólicas $(\mathrm{p}=0,000)$. Conclusión: El estudio permitió conocer el perfil de uso de fármacos continuos y las variables asociadas, estos datos son fundamentales para proponer medidas que mejoren la efectividad y seguridad de la quimioterapia y otros fármacos asociados.
\end{abstract}

Palabras clave: Medicamentos; Cáncer; Enfermedades crónicas; Paciente.

\title{
1. Introdução
}

As doenças crônicas não transmissíveis (DCNT) são as principais responsáveis pelo adoecimento e óbito da população no mundo. Em 2008, aproximadamente 36 milhões de mortes ocorreram em consequência de DCNT, com destaque para doenças cardiovasculares (48\%) e câncer (21\%) (Organização Mundial da Saúde [OMS], 2013). No Brasil, as DCNT aumentam em ritmo acelerado e se constituem em um problema de saúde pública (Schmidt et al., 2011).

O câncer, considerado uma DCNT, cuja estimativa mundial mostrou que, em 2018, ocorreram 18,1 milhões de novos casos e 9,6 milhões de mortes em decorrência da doença, sendo mais incidente a nível mundial o câncer de pulmão, mama, intestino e próstata, quando comparado a outros tipos (Bray et al., 2018). O aumento da ocorrência de câncer é decorrente do envelhecimento da população e da elevada prevalência dos fatores de risco, ou seja, devido ao estilo de vida contemporâneo que pode favorecer a formação de tumores (Torre et al., 2012). O tratamento do câncer pode ser realizado por meio de cirurgia, quimioterapia, hormonioterapia e, em alguns casos, transplante de medula óssea Santos et al. (2011) ou ainda pela utilização de diferentes classes de medicamentos, como aminoglicosídeos, agentes antineoplásicos, antibióticos, anti-inflamatórios não esteroidais, diuréticos, entre outros (Oliveira et al., 2016).

O uso de medicamentos na população brasileira está aumentando, a prevalência de utilização de medicamentos no Brasil é de $49 \%$ a 56,9\% (Carvalho et al., 2003). Estudo de Bertoldi et al. (2016) apontou prevalência do uso de medicamentos para doenças crônicas no Brasil entre 2013 e 2014 de 24,3\%, 18,7\% nos indivíduos do sexo masculino e 29,4\% no sexo feminino. Observou-se, no mesmo, estudo aumento da prevalência de medicamentos crônicos com o aumento da idade.

$\mathrm{O}$ uso adequado de medicamentos possibilita o controle das doenças, a redução da morbimortalidade e a melhora da qualidade de vida dos usuários Malta \& Silva (2013) quando utilizados de forma correta. No entanto, Mendes (2011) salienta que a dificuldade de acesso aos serviços de saúde e aos medicamentos faz com que as pessoas não tenham um diagnóstico correto ou não os utilizem de forma adequada, o que gera ineficácia dos fármacos pela não adesão à terapia.

A falta de adesão e o uso irracional de medicamentos pode piorar o quadro clínico dos indivíduos acometidos por condições crônicas. No Brasil, entre 2013 e 2017, os medicamentos foram responsáveis por 131.868 casos de intoxicação registrados e 366 casos de morte por estes eventos (Sereno et al., 2020). Os indivíduos com DCNT usam mais medicamentos, portanto, são mais vulneráveis aos problemas relacionados ao uso dos mesmos (World Health Organization, 2013). Não foram encontrados estudos que correlacionam especificamente o uso de medicamentos contínuos com a quimioterapia em pacientes oncológicos até o presente momento. 
Diante disso, o objetivo deste trabalho foi descrever o perfil de tratamento de pacientes oncológicos que fazem uso de medicamentos contínuos, assistidos em um CACON.

\section{Metodologia}

Trata-se de um estudo transversal, quantitativo e analítico. A amostra constituiu-se de pacientes com diagnóstico de câncer, inseridos para iniciar o tratamento oncológico junto ao Centro de Alta Complexidade (CACON), de um hospital porte IV, do noroeste do Estado do Rio Grande do Sul, no período de agosto de 2018 a janeiro de 2019.

A coleta de dados consistiu na aplicação de um instrumento com variáveis de identificação, sociodemográficas, clínicas, hábitos de vida e uso de medicamentos. Os dados referentes à comorbidades, hábitos de vida e uso de medicamentos foram auto relatados. Foi considerado medicamento de uso contínuo o utilizado há pelo menos 6 meses. Os entrevistados foram convidados a participar da pesquisa de forma espontânea e assinarem o Termo de Consentimento Livre e Esclarecido (TCLE).

Na pesquisa, foram incluídos pacientes maiores de 18 anos de idade, diagnosticados com qualquer tipo de câncer, que não deram início ao tratamento oncológico e que aceitaram participar da pesquisa. Foram excluídos da pesquisa pacientes que apresentaram dificuldade ou incapacidade para compreender as questões do instrumento de pesquisa ou que não aceitaram participar da mesma e aqueles que já iniciaram tratamento oncológico no local.

Os dados foram avaliados através da análise descritiva simples com média e desvio padrão, com o auxílio do software Statistical Pakage for Social Science (SPSS) versão 20.0. Foi realizado teste de Qui-quadrado $\left(\chi^{2}, \mathrm{p}>0,05\right)$ para avaliar associação entre uso de medicamentos e variáveis sociodemográficas. A classificação farmacológica dos medicamentos foi realizada de acordo com a Classificação Anatômica Química (ATC) (13) do WHO Collaborating Centre for Drug Statistics Methodology.

Esta pesquisa foi aprovada pelo Comitê de Ética em Pesquisa da Universidade de Cruz Alta sob n ${ }^{\circ} 2.626 .873$ e conduzida de acordo com os aspectos éticos preconizados pela Resolução CNS n 466/2012.

\section{Resultados}

A amostra foi constituída por 270 pacientes oncológicos, destes, 53\% era do sexo feminino e 58,1\% com mais de 60 anos de idade (Tabela 1).

Dos entrevistados, $74,1 \%$ fazem uso de medicamentos contínuos, a maioria do sexo feminino e vivem acompanhados. Com relação à escolaridade, o maior percentual $(59,8 \%)$ dos entrevistados que faz uso de medicamentos cursou o ensino fundamental completo, conforme Tabela 1. 
Tabela 1: Dados sociodemográficos de pacientes oncológicos em tratamento com medicamentos contínuos do município de Ijuí/RS. n=270. 2019.

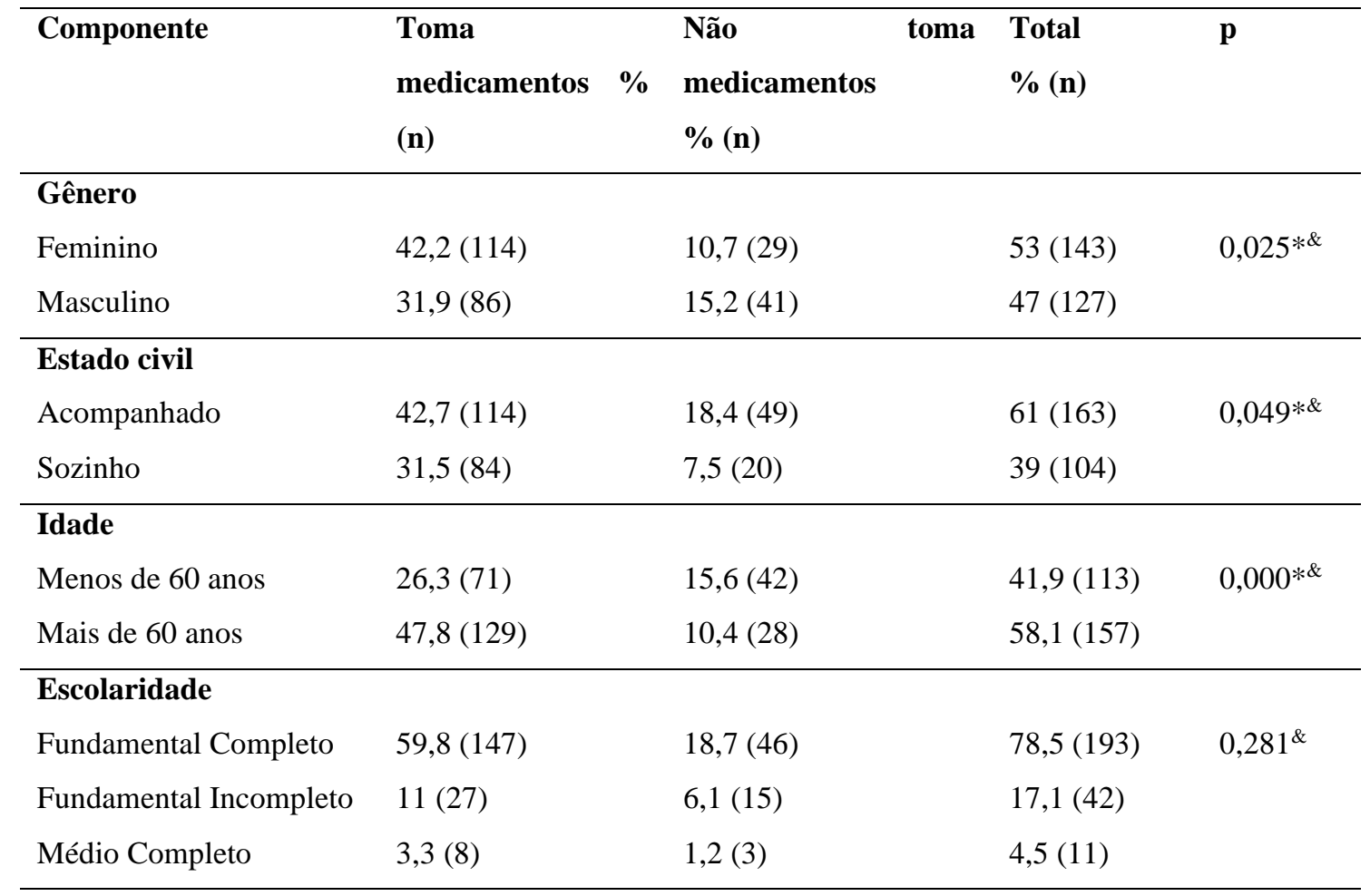

* p <0,05 = significância estatística; \& teste de Qui-quadrado. Fonte: Autores.

Verificou-se associação entre uso de medicamentos e: sexo feminino ( $\mathrm{p}=0,025)$; ser casado ou ter acompanhante ( $\mathrm{p}=0,049)$; e possuir mais de 60 anos $(\mathrm{p}=0,000)$.

O grupo de medicamentos mais utilizado pelos pacientes oncológicos em tratamento com medicamentos contínuos foi do aparelho cardiovascular (69,56\%). Os medicamentos mais citados pelos pacientes nesse grupo foram losartana (12,2\%), enalapril $(10,0 \%)$ e hidroclorotiazida $(8,88 \%)$, conforme Tabela 2.

Outro grupo em destaque na pesquisa foi o do sistema nervoso (28,87\%), com maior frequência de amitriptilina $(2,22 \%)$, escitalopram (2,22\%) e fluoxetina (2,22\%). Dos entrevistados, 4,07\% utilizam de forma contínua o medicamento ácido acetilsalicílico, inserido no grupo de aparelho digestivo e metabolismo. No referido grupo, o omeprazol também foi citado pelos pacientes em tratamento de câncer com percentual de 3,70\% (Tabela 2).

Tabela 2: Medicamentos contínuos utilizados por pacientes oncológicos no município de Ijuí/RS conforme o primeiro e quinto nível da Anatomical Therapeutic Chemical (ATC). n=270. 2019.

\begin{tabular}{llll}
\hline Grupo & Substância química & N & \% \\
\hline & Ácido acetilsalicílico & 11 & 4,07 \\
& Cálcio & 2 & 0,74 \\
Aparelho digestivo e metabolismo & Glibenclamida & 4 & 1,48 \\
& Metformina & 8 & 2,96 \\
& Omeprazol & 10 & 3,70 \\
& Polivitamínico & 5 & 1,85 \\
\hline
\end{tabular}


Research, Society and Development, v. 10, n. 6, e34010615481, 2021

(CC BY 4.0) | ISSN 2525-3409 | DOI: http://dx.doi.org/10.33448/rsd-v10i6.15481

\begin{tabular}{|c|c|c|c|}
\hline \multirow{3}{*}{ Sangue e órgãos hematopoiéticos } & Clopidogrel & 2 & 0,74 \\
\hline & Varfarina & 2 & 0,74 \\
\hline & Outros & 3 & 1,11 \\
\hline \multirow{13}{*}{ Aparelho cardiovascular } & Atenolol & 18 & 6,66 \\
\hline & Captopril & 20 & 7,40 \\
\hline & Doxazosina & 3 & 1,11 \\
\hline & Enalapril & 27 & 10,0 \\
\hline & Espironolactona & 3 & 1,11 \\
\hline & Furosemida & 6 & 2,22 \\
\hline & Hidroclorotiazida & 24 & 8,88 \\
\hline & Losartana & 33 & 12,2 \\
\hline & Ômega 3 & 3 & 1,11 \\
\hline & Propranolol & 4 & 1,48 \\
\hline & Sinvastatina & 19 & 7,03 \\
\hline & Succinato de metoprolol & 4 & 1,48 \\
\hline & Outros & 24 & 8,88 \\
\hline Medicamentos dermatológicos & Mepiramina & 1 & 0,37 \\
\hline \multirow[t]{2}{*}{ Aparelho gênito-urinário e hormônios sexuais } & Levonorgestrel/etinilestradiol & 2 & 0,74 \\
\hline & Outros & 3 & 1,11 \\
\hline $\begin{array}{l}\text { Preparações hormonais sistêmicas, excluindo } \\
\text { hormônios sexuais e insulinas }\end{array}$ & Levotiroxina & 6 & 2,22 \\
\hline Anti-infecciosos gerais para uso sistêmico & Coquetel HIV & 1 & 0,37 \\
\hline \multirow[t]{2}{*}{ Agente antineoplásico e imunomoduladores } & Tacrolimo & 1 & 0,37 \\
\hline & Micofenolato & 1 & 0,37 \\
\hline \multirow{2}{*}{ Sistema músculo-esquelético } & Condroitina/glicosamina & 3 & 1,11 \\
\hline & Diclofenaco & 1 & 0,37 \\
\hline \multirow{12}{*}{ Sistema nervoso } & Amitriptilina & 6 & 2,22 \\
\hline & Betaistina & 3 & 1,11 \\
\hline & Clonazepam & 5 & 1,85 \\
\hline & Diazepam & 5 & 1,85 \\
\hline & Escitalopram & 6 & 2,22 \\
\hline & Fenitoína & 5 & 1,85 \\
\hline & Fluoxetina & 6 & 2,22 \\
\hline & Paracetamol & 3 & 1,11 \\
\hline & Risperidona & 3 & 1,11 \\
\hline & Sertralina & 4 & 1,48 \\
\hline & Venlafaxina & 3 & 1,11 \\
\hline & Outros & 29 & 10,74 \\
\hline \multirow{4}{*}{ Aparelho respiratório } & Formoterol/budesonida & 6 & 2,22 \\
\hline & Salbutamol & 3 & 1,11 \\
\hline & Bamifilina & 2 & 0,74 \\
\hline & Outros & 4 & 1,48 \\
\hline
\end{tabular}

Fonte: Autores. 
Quando perguntados sobre as doenças coexistentes, 48,7\% dos participantes afirmaram ter hipertensão arterial sistêmica e utilizar os medicamentos para controle da doença. Dos entrevistados, 40 deles autorrelataram ter diabetes mellitus e destes, 13,3\% utilizam os medicamentos contínuos. Diferentemente dos pacientes que tiveram infarto agudo do miocárdio, os quais todos mencionaram fazer uso de medicamentos para controle da doença. Entre os pacientes que faziam uso de medicamentos, verificou-se que $24,1 \%$ tinham depressão (Tabela 3 ).

A maioria dos pacientes oncológicos entrevistados mencionou que não praticava exercícios físicos $(72,6 \%)$, e entre estes, 142 (72,5\%) utilizavam medicamentos, sem associação estatística. Com relação ao fumo, a maioria dos entrevistados não fumava e destes $78 \%$ utilizava medicamentos. No que se refere ao consumo de bebidas alcoólicas, 45,6\% dos pacientes oncológicos não utilizam bebidas alcoólicas e faziam uso de medicamentos, conforme Tabela 3.

Tabela 3: Doenças e fatores de risco autorrelatado por pacientes oncológicos em tratamento com medicamentos contínuos do município de Ijuí/RS. n=270. 2019.

\begin{tabular}{|c|c|c|c|c|}
\hline Componente & $\begin{array}{l}\text { Toma } \\
\text { medicamentos \% (n) }\end{array}$ & $\begin{array}{l}\text { Não toma medicamentos } \\
\%(\mathbf{n})\end{array}$ & $\begin{array}{l}\text { Total } \\
\%(n)\end{array}$ & $\mathbf{p}$ \\
\hline \multicolumn{5}{|c|}{ Hipertensão Arterial Sistêmica } \\
\hline Sim & $48,7(131)$ & $1,5(4)$ & $50,2(135)$ & $0,000 *$ \\
\hline Não & $25,3(68)$ & $24,5(66)$ & $49,8(134)$ & \\
\hline \multicolumn{5}{|l|}{ Diabetes Mellitus } \\
\hline Sim & $13,3(36)$ & $1,5(4)$ & $14,8(40)$ & $0,013^{*}$ \\
\hline Não & $60,7(164)$ & $24,4(66)$ & $85,2(230)$ & \\
\hline \multicolumn{5}{|c|}{ Colesterol e triglicerídeos } \\
\hline Sim & $20,7(56)$ & $4,4(12)$ & $25,2(68)$ & 0,072 \\
\hline Não & $53,3(144)$ & $21,5(58)$ & $74,8(202)$ & \\
\hline \multicolumn{5}{|c|}{ Infarto Agudo do Miocárdio } \\
\hline Sim & $4,1(11)$ & $0,0(0)$ & $4,1(11)$ & $0,045^{*}$ \\
\hline Não & $70(189)$ & $25,9(70)$ & $95,9(259)$ & \\
\hline \multicolumn{5}{|l|}{ Depressão } \\
\hline Sim & $21,5(58)$ & $2,6(7)$ & $24,1(65)$ & $0,001 *$ \\
\hline Não & $52,6(142)$ & $23,3(63)$ & $75,9(205)$ & \\
\hline \multicolumn{5}{|l|}{ Exercícios físicos } \\
\hline Sim & $21,5(58)$ & $5,9(16)$ & $27,4(74)$ & 0,321 \\
\hline Não & $52,6(142)$ & $20,0(54)$ & $72,6(196)$ & \\
\hline \multicolumn{5}{|l|}{ Fuma } \\
\hline Sim & $11,5(31)$ & $5,9(16)$ & $17,4(47)$ & 0,189 \\
\hline Não & $44,8(121)$ & $12,6(34)$ & $57,4(155)$ & \\
\hline Ex fumantes & $17,8(48)$ & $7,4(20)$ & $25,2(68)$ & \\
\hline \multicolumn{5}{|l|}{ Drogas } \\
\hline Sim & $0,4(1)$ & $0,0(0)$ & $0,4(1)$ & 0,871 \\
\hline Não & $72,2(195)$ & $25,6(69)$ & $97,8(264)$ & \\
\hline Não responderam & & & $1,8(5)$ & \\
\hline \multicolumn{5}{|l|}{ Bebidas alcóolicas } \\
\hline Sim & $16,7(45)$ & $11,9(32)$ & $28,5(77)$ & $0,000 *$ \\
\hline Não & $45,6(123)$ & $9,3(25)$ & $54,8(148)$ & \\
\hline Não responderam & & & $16,7(45)$ & \\
\hline
\end{tabular}


Fonte: Autores.

Foi verificada associação entre usar medicamentos contínuos e autorrelato de: hipertensão ( $\mathrm{p}=0,000)$; diabetes mellitus ( $\mathrm{p}=0,013)$; infarto agudo do miocárdio $(\mathrm{p}=0,045)$; depressão $(\mathrm{p}=0,001)$ e uso de bebidas alcóolicas $(\mathrm{p}=0,000)$.

Dos entrevistados, os cânceres com maior prevalência foram: mama $(14,4 \%)$, seguido de traqueia $(5,9 \%)$ e colo de útero $(5,9 \%)$

\section{Discussão}

Neste estudo, um pouco mais da metade dos pacientes oncológicos em tratamento com medicamentos contínuos eram mulheres, com mais de 60 anos de idade. Mendes et al. (2014) em estudo com indivíduos com doenças crônicas não oncológicas verificou que 55,5\% dos mesmos eram mulheres e a maioria idosas. De acordo com a literatura, mulheres têm mais preocupação em relação à saúde do que os homens, procuram os serviços médicos mais precocemente e com mais frequência (Arrais et al., 2005).

No presente estudo, verificou-se associação entre o uso de medicamentos e idade superior a 60 anos. Secoli (2010) em seu estudo identificou o aumento do consumo de medicamentos entre os idosos em consequência da prevalência de doenças crônicas e das sequelas que acompanham o avançar da idade. Assim, os casos de câncer acometem mais pacientes idosos, que, como exposto, são polimedicados em função das doenças crônicas.

A maioria dos entrevistados tem ensino fundamental completo e reside com familiares. Já no estudo de Silva et al. (2012), com 384 idosos usuários de medicamentos contínuos, foi constatado que 54,4\% dos entrevistados possuíam ensino fundamental incompleto e moravam com até três pessoas (63,5\%). Em outra pesquisa com 360 idosos, constatou-se que $78 \%$ eram do sexo feminino, $83 \%$ residiam sozinhos e 85,8\% tinham até quatro anos de estudo (Carneiro et al., 2017) (18). A baixa escolaridade dificulta o entendimento e a adesão ao tratamento farmacológico, o que pode comprometer os resultados da quimioterapia de pacientes que fazem uso de medicamentos associados ao tratamento para o câncer.

Não foram encontrados outros estudos na literatura que abordassem o uso de medicamentos contínuos e características sociodemográficas em pacientes oncológicos. Contudo, os resultados desta pesquisa demonstram necessidade de cuidado, em especial, com mulheres, idosas e que residem com acompanhantes.

Conforme a Tabela 2, os medicamentos losartana, enalapril e hidroclorotiazida foram os mais citados pelos entrevistados. No estudo de Sales et al. (2019) sobre polifarmácia em idosos oncológicos, o autor constatou que os medicamentos mais utilizados pelos pacientes foram losartana $(26,4 \%)$, hidroclorotiazida $(23,2)$, omeprazol $(15,1 \%)$, metformina $(13,0 \%)$ e sinvastatina $(12,6 \%)$.

Sobre estes medicamentos supracitados verificou-se que a losartana interage com mitotano e axitinib, utilizados para a quimioterapia. A hidroclorotiazida interage com ciclofosfamida e fluorouracil também antineoplásicos (Medscape, 2020). Esta interação é relevante, pois os diuréticos tiazídicos podem aumentar os efeitos mielossupressores dos antineoplásicos. Sugere-se que, caso a terapia concomitante for necessária, é preciso acompanhar a mielossupressão (Micromedex, 2020).

Outro grupo que se destacou no estudo foram os medicamentos do sistema nervoso, com destaque para a amitriptilina, escitalopram e fluoxetina. Os quimioterápicos (nilotinibe, pazopanibe, sorafenibe e vemurafenibe) interagem com o antidepressivo tricíclico (Micromedex, 2020). O escitalopram interage com o medicamento tamoxifeno, nilotinibe, lapatinibe e sunitinibe, e ainda, a fluoxetina interage com tamoxifeno, abiraterona, lapatinibe, nilotinibe, sorafenibe e sunitinibe (Micromedex, 2020; Santana, 2028). A interação entre tamoxifeno e antidepressivos reduz o nível do metabólito ativo e interage significativamente na eficácia clínica do tratamento (Breitbart, 2010). Um estudo observacional subsequente de mulheres tratadas com tamoxifeno demonstrou baixas concentrações séricas do metabólito ativo em pacientes tratadas concomitantemente com 
inibidores potentes do CYP2D6, tais como paroxetina e fluoxetina, e níveis intermediários do metabólito em mulheres tratadas com inibidores moderados e fracos, como sertralina e citalopram (Appiani et al., 2011).

Esses dados demonstram que existem potenciais interações entre os medicamentos contínuos em uso domiciliar e os específicos para o tratamento do câncer, o que requer a necessidade de um cuidado integral ao paciente. Nesse sentido, é importante avaliar a relação risco-benefício de cada associação medicamentosa e adotar medidas que possam reduzir os efeitos negativos das interações.

Verificou-se que pacientes com hipertensão arterial sistêmica, usaram maior número de medicamentos do que aqueles que não relataram tal problema, conforme Tabela 3. Como abordado anteriormente, pacientes polimedicados têm mais riscos de interações medicamentosas e necessitam de mais cuidado quando em tratamento com medicamentos antineoplásicos. Nesse contexto, são necessárias medidas para amenizar os riscos das interações medicamentosas, através do acompanhamento farmacoterapêutico diferenciado.

Entre os tipos de câncer verifica-se o uso de medicamentos superior a $70 \%$ em todos os casos, valor superior à porcentagem geral apresentada no estudo. Este fator demonstra que, nesses tipos de câncer, ocorre maior uso de medicamentos, o que pode estar associado com sexo, idade, comorbidades, e outros fatores de risco, conforme descritos. Também se verifica que tais tipos de câncer são aqueles que necessitam de acompanhamento mais próximo dos pacientes para evitar interações medicamentosas, iatrogenias e riscos relacionados à farmacoterapia que podem comprometer o resultado clínico e a qualidade de vida do paciente. Destaca-se que a menor efetividade de um medicamento quimioterápico em função de interação medicamentosa pode gerar ineficácia terapêutica, progressão da doença e risco ao paciente. Por isso evidencia-se a necessidade de avaliar o paciente como um todo e não apenas o câncer, visando reduzir riscos e melhorar a sobrevida.

O farmacêutico tem papel fundamental no cuidado ao paciente oncológico e usuário de medicamentos contínuos, pois este auxilia na conciliação dos medicamentos, busca reduzir as interações medicamentosas e os eventos adversos pelo uso concomitante de dois ou mais medicamentos, e orienta quanto ao uso correto dos medicamentos contínuos durante o tratamento com antineoplásicos. Além disso, o farmacêutico prioriza a adesão ao tratamento e o consumo racional dos medicamentos prescritos.

\section{Conclusão}

É importante conhecer o perfil de pacientes oncológicos quanto ao uso de medicamentos contínuos para garantir a segurança dos pacientes e a efetividade dos tratamentos. Observou-se no estudo associação entre idade dos pacientes e o uso de medicamentos e também interações medicamentosas entre os medicamentos de uso contínuo com os antineoplásicos. Estes dados reforçam a necessidade de acompanhamento farmacêutico, de maneira a garantir o tratamento adequado ao paciente. Não foi avaliado o medicamento oncológico que cada paciente fazia uso, tratando-se de uma limitação do estudo.

\section{Referências}

Appiani, F., Brendan, T. C., Munoz, C., \& Trecco, J. Tamoxifen-SSIRs interaction: Clinical manifestations of inhibition and lack of inhibition os CYP2D6. Ann Oncol. 2011;23(2):152-153.

Arrais PSD, Brito LL, Barreto ML, Coelho HL. Prevalência e fatores determinantes do consumo de medicamentos no Município de Fortaleza, Ceará, Brasil. Cad Saúde Pública. 2005;21(6):1737-46.

Bertoldi, A. D., Pizzol, T. S. D., Ramos, L. R., Mengue, S. S., Luiza, V. L., Tavares, N. U. L., Farias, M. R., Oliveira, M. A., \& Arrais, P. S. D. (2016).Perfil sociodemográfico dos usuários de medicamentos no Brasil: resultado do PNAUM 2014. Revista Saúde Pública, 50(2).

Bray, F., Ferlay, J., Soerjomataram, I., Siegel, R. L., Torre, L. A., \& Jemal, A. (2018). Global cancer statistics 2018: GLOBOCAN estimates of incidence and mortality worldwide for 36 cancers in 185 countries. CA: a cancer journal for clinicians, 68(6), 394-424. https://doi.org/10.3322/caac.21492.

Breitbart W. Do antidepressants reduce the effectiveness of tamoxifen? Psychooncology. 2010;20(1):1-4. 
Carneiro, J. A., Cardoso, R. R., Durães, M. S., Guedes, M. C. A., Santos, F. L., Costa, F. M., et al. Frailty in the elderly: prevalence and associated factors. Rev Bras Enferm. 70(4):747-52.

Carvalho, M. F., Pascom, A. N. P., Souza Júnior, P. R. B., Damacena, G. N., \& Szwarcwald, C. L.(2005). Utilization of medicines by the Brazilian population, 2003. Cad Saúde Pública, 21(1)100-8

Malta, D. C., \& Silva Júnior, J. B. (2013). O plano de ações estratégicas para o enfrentamento das doenças crônicas não transmissíveis no Brasil e a definição das metas globais para o enfrentamento dessas doenças até 2025: uma revisão. Epidemiol Serv Saúde, 22(1)151-64.

Medscape. [Internet]. Drug Interaction Checker. https://reference.medscape.com/drug-interactionchecker .

Mendes, E. V. (2011). As redes de atenção à saúde. Brasília-DF: Organização Pan-Americana da Saúde, 549.

Mendes, L. V., Emmerick, I. C. M., \& Luiza, V. L. (2014). Uso de medicamentos entre portadores de doenças crônicas: um estudo observacional no estado do Espírito Santo. Rev. Bras. Farm, 95(2):732-947.

Micromedex Healthcare. [Internet]. Drugdex System. Greenwood Village: Series 20: Interactions, 2011. http://www.biblioteca.ufrgs.br/bases.

Micromedex. [Internet] Greenwood Village, Colorado, USA; c1974-2012. http://www.thomsonhc.com.

Oliveira, F. O., Oliveira, C. S., Andrade, J. S., Santos, T. F. C., \& Oliveira Barreto, A. C.(2016). Cancer treatment in determination of hearing loss. Rev Bras Otorrinolaringol, 82(1):65-9.

Organização Mundial da Saúde. (2013). Doenças crônicas não transmissíveis. https://www.paho.org/bra/index.php?option=com_conte nt\&view=article\&id=4766:doencas-cronicas-nao-transmissiveis-causam-16-milhões-de-mortes-prematuras-todos-os-anos \&Itemid=839.

Sales, J. T. O. L., Nova, T. C. A., Lopes, I. M., Wanderley, C. M. S., Bezerra, M.R., Farias, I. M. N. \& Mello, M. J. G. (2019). Epidemiologia da polifarmácia em idosos oncológicos: estudo de coorte prospectivo. http://higia.imip.org.br/handle/123456789/358.

Santana RM. Abordagem teórica sobre o uso de medicamentos antidepressivos por pacientes em terapia antineoplásica. [Internet] Governador Mangabeira/BA, 2018http://131.0.244.66:8082/jspui/bitstream/123456789/739/1/Rogelly\%20Malta\%20de\%20Santana.pdf.

Santos, M. A., Prado, M. A. S., Panobianco, M. S., \& Almeida, A. M. (2011). Grupo de apoio a mulheres mastectomizadas: Cuidando das dimensões subjetivas do adoecer. Revista da SPAGESP, 12(2), 27-33.

Schmidt, M. I., Duncan, B. B., Azevedo e Silva, G., Menezes, A. M., Monteiro, C.A., Barreto, S., Chor, D., \& Menezes, P.R. (2011). Chronic non-communicable diseases in Brazil: burden and current challenges. The Lancet, 377,1949-61.

Secoli, S. R. (2010). Polifarmácia: interações e reações adversas no uso de medicamentos por idosos. Rev. Bras. Enferm, 63(1):136-140.

Sereno, V. M. B., Silva, A. S., \& Silva, C. V. (2020) Perfil epidemiológico das intoxicações por medicamentos no Brasil entre os anos de 2013 a 2017. Brazilian Journal of Development, 6(6), 33892-33903. https://doi.org/10.34117/bjdv6n6-079.

Silva, G. O. B., Gondim, A. P. S., Monteiro, M. P., Frota, M. A., \& Meneses, A. L. L. (2012). Uso de medicamentos contínuos e fatores associados em idosos de Quixadá, Ceará. Rev Bras. Epidemol, 15(2):386-95.

Torre, L. A., Bray, F., Siegel, R. L., Ferlay, J., Lortet-Tieulent, J., \& Jemal, A. (2015). Global cancer statistics, 2012. CA: a cancer journal for clinicians, 65(2), 87-108. https://doi.org/10.3322/caac.21262.

World Health Organization. (2003). Adherence to long-term therapies: evidence for action. Geneva: World Health Organization. https://www.who.int/chp/knowledge/publications/adherence_report/en/.

World Health Organization. (2016) Anatomical Therapeutic Chemical. http://www.whocc.no/atc_ddd_index/. 